\title{
Democracia paritaria en Latinoamérica: la situación de jure y de facto de la participación política de las mujeres en el contexto boliviano**
}

\section{Joint democracy in Latin America: The de jure and de facto situation of the political participation of women in the Bolivian context}

RESUMEN

El contexto histórico del desarrollo de los derechos políticos de las mujeres refleja una constante discriminación a favor del género masculino, por ello, y en buena medida gracias a una serie de conquistas significativas de las mismas mujeres, se van adoptando diversos instrumentos internacionales específicos de protección que permitan lograr cierta igualdad de oportunidades en derechos de participación política. En el caso del contexto boliviano, en el marco de la Constitución Política del Estado y de diferentes leyes de desarrollo constitucional, se ha logrado consolidar a Bolivia como el único país de América Latina en lograr resultados de paridad en todos los niveles estatales. No obstante, y sin perder objetividad, el trabajo refleja también la sistemática violación de derechos de participación política de autoridades

Abogado de la Universidad Mayor de San Andrés, diplomado en Educación Superior e Investigación de la Unidad de Post Grado y Relaciones Internacionales de la Facultad de Derecho y Ciencias Políticas - UMSA; diplomado en Argumentación Jurídica de la Universidad Privada Franz Tamayo (UNIFRANz); máster en Derecho Constitucional y Derecho Procesal Constitucional de la Universidad Andina Simón Bolívar (UASB). Miembro asociado de la organización académica judicial internacional E-Justicia Latinoamérica de Buenos Aires, Argentina. Actualmente profesor de las asignaturas Derecho Procesal Constitucional y Derechos Humanos de la Universidad Privada Franz Tamayo, Sede la Paz. Contacto: oscarbarrientosjimenez@gmail.com

** El presente trabajo fue expuesto en la Primera Convención Mundial de Derechos Fundamentales y Garantías Constitucionales en honor al profesor Dr. h.c.mult. Robert Alexy, realizada en el año 2016 en Lima, Perú.

Recibido el 13 de febrero de 2017, aprobado el 15 de octubre de 2017.

Para citar el artículo: BarRIEnTos JimÉnEZ, Ó. G. Democracia paritaria en Latinoamérica: la situación de jure y de facto de la participación política de las mujeres en el contexto boliviano. Derecho del Estado n. ${ }^{\circ}$ 40, Universidad Externado de Colombia, enero-junio de 2018, pp. 87112. DOI: https://doi.org/10.18601/01229893.n40.04 
municipales electas. A continuación se analiza la aplicación de la primera ley específica de acoso y violencia política en Latinoamérica. Las conclusiones muestran que la construcción de una democracia paritaria plena contribuye a la legitimidad democrática, el buen gobierno y el desarrollo sostenible de cualquier país.

PALABRAS CLAVE

Discriminación, derechos de participación política, género, democracia paritaria, acoso y violencia política, Bolivia.

\section{ABSTRACT}

The historical context of the development of women's political rights reflects a constant discrimination in favor of the masculine gender, and to a large extent thanks to a series of significant achievements of the same women, a number of specific international protection instruments are adopted. Allow for some equality of opportunity in political participation rights. In the case of the Bolivian context within the framework of the Political Constitution of the State and different constitutional development laws, Bolivia has been consolidated as the only country in Latin America to achieve parity results at all levels. Nevertheless, and without losing objectivity, the work also reflects the systematic violation of the rights of political participation of municipal elected officials. Analyzing below the application of the first specific law of harassment and political violence in Latin America. Final considerations will demonstrate that only building a full parity democracy will contribute to the democratic legitimacy, good governance and sustainable development of any country.

KEYWORDS

Discrimination, political participation rights, gender, parity democracy, harassment and political violence, Bolivia.

SUMARIO

Introducción. 1. Principales antecedentes normativos internacionales de protección de derechos civiles y políticos de las mujeres. 2. La situación de jure de la participación política de las mujeres en el contexto boliviano. 3. La situación de facto de la participación política de las mujeres en el contexto boliviano. 3.1. La participación política de las mujeres a nivel nacional. 3.2. La participación política de las mujeres a nivel departamental. 3.3. La participación política de las mujeres a nivel municipal. 4. Acoso y violencia 
política contra las mujeres. 4.1. Juana Quispe Apaza, uno de los casos más extremos de acoso y violencia política. 5. La primera ley específica sobre acoso y violencia política de América Latina y sus claroscuros. 5.1. El Decreto Supremo n. ${ }^{\circ} 2935$ de 5 de octubre de 2016. Conclusión. Referencias.

\section{INTRODUCCIÓN}

\section{Como tan acertadamente afirma Rosalía Camacho:}

Si bien el nacimiento de los derechos humanos significó un importante acontecimiento en la historia de la humanidad, ello no aseguró a las mujeres que sus necesidades, experiencias de vida y puntos de vista pasarían a formar parte de la teoría y práctica de los mismos. Al ser creados tomando como parámetro o modelo de la humanidad al hombre varón, los derechos humanos excluyeron a las mujeres y no tomaron en cuenta sus circunstancias específicas, ni consideraron que las violaciones a sus derechos eran violaciones a los derechos humanos. Hasta hoy en día, la historia demuestra que lo humano sigue siendo entendido, en general, como sinónimo de lo masculino. El sistema de los derechos humanos no ha escapado a esa visión androcéntrica, es decir, a tomar al hombre varón como el centro a partir del cual se ha desarrollado el pensamiento humano. El androcentrismo es una de las manifestaciones más comunes del sexismo que ha dado como resultado la existencia y reproducción de la discriminación, la desigualdad y los prejuicios en contra de las mujeres ${ }^{1}$.

Lo anterior explica la lucha por el ejercicio pleno de los derechos de las mujeres en relación con su ciudadanía y su participación activa en la esfera política, reflejo una discriminación histórica a favor del género masculino. En efecto, aún

... persisten en la región factores estructurales que todavía impiden o limitan el pleno ejercicio de los derechos políticos de las mujeres. Ello se refleja en las actitudes culturales basadas en modelos patriarcales, estereotipos sexistas y roles tradicionales de hombres y mujeres, en el deficitario empoderamiento político y económico de las mujeres o en los dramáticos datos sobre violencia de género. Igualmente, se observa el sesgo machista de los medios de comunicación, de los partidos políticos, o los problemas de conciliación entre la vida familiar y profesional (que afectan mayoritariamente a las mujeres) ${ }^{2}$.

1 Camacho, R. Acercándonos a los instrumentos internacionales de protección de los derechos humanos de las mujeres. 1. a reimpr. San José: Instituto Interamericano de Derechos Humanos, 2004, 16-17.

2 ONu Mujeres y Parlamento Latinoamericano y Caribeño. Norma Marco para consolidar la democracia paritaria, 5. [En línea]. [Consulta: 7 de agosto de 2016]. Disponible en: http:// www.parlatino.org/pdf/leyes_marcos/leyes/consolidar-democracia-paritaria-pma-27-nov-2015. pdf 
En este contexto, un objetivo del presente trabajo es reflejar el desarrollo progresivo de los diferentes instrumentos internacionales de protección de derechos civiles y políticos de las mujeres en procura de lograr una necesaria e importante igualdad de oportunidades. Un segundo propósito es evidenciar que pese a los avances normativos y las cuotas de género que permitieron resultados de paridad, las mujeres aún continúan en situación de desigualdad, en especial para acceder o ejercer de manera efectiva cargos políticos o de alto nivel de decisión, aspectos que reflejamos al analizar integralmente la situación de los derechos políticos de las mujeres en el contexto boliviano.

\section{PRINCIPALES ANTECEDENTES NORMATIVOS INTERNACIONALES DE PROTECCIÓN DE DERECHOS CIVILES Y POLÍTICOS DE LAS MUJERES}

La Declaración de los Derechos del Hombre y del Ciudadano fue el documento fundamental de la Revolución Francesa de 1789. En ella se consagraron las ideas de igualdad, de libertad, de soberanía popular, de limitación al poder estatal, del derecho a controlar a los gobernantes ${ }^{3}$. En cuanto a los derechos de las mujeres, los pensadores más influyentes mostraron diferencias de grado al atribuirles inferioridad con respecto a los hombres. Unos las consideraron seres naturalmente inferiores, con cuerpos y mentes hechos exclusivamente para tener hijos y estar en la casa, solo capaces de atender los intereses inmediatos de la familia. No faltaron quienes le atribuyeran un funcionamiento cerebral que las hacía ser apasionadas. Pero todos coincidieron en cuanto a que no eran aptas para participar en la vida política, y menos aún para decidir, legislar o representar los intereses de la ciudadanía; los iguales eran los hombres ${ }^{4}$.

Casi más de dos siglos después, la Carta de las Naciones Unidas, que se firmó el 26 de junio de 1945 en San Francisco, en su nota introductoria reafirmo la "fe en los derechos fundamentales, en la dignidad y el valor de toda persona humana, y en los derechos iguales de hombres y mujeres", a la vez que estableció que toda persona, con independencia de de su sexo, goza de los derechos humanos.

El año 1948, en la Ix Conferencia Internacional Americana realizada en Bogotá, la Organización de Estados Americanos (oEA) aprobó la Declaración Americana de los Derechos y Deberes del Hombre, la cual, no obstante su

3 Olympe de Gouges, cuyo nombre real fue Marie Gouze, en 1791 escribió la Declaración de los Derechos de la Mujer y la Ciudadana. Denuncia la negación de los derechos de las mujeres contenidos en la Declaración de Derechos del Hombre y del Ciudadano y propone la emancipación femenina en el sentido de la igualdad de derechos o la equiparación jurídica y legal de las mujeres en relación con los varones. Fue guillotinada el 3 de noviembre de 1793.

4 Instituto Interamericano de Derechos Humanos. Aula Virtual Interamericana. Curso auto formativo. Recursos para incorporar los derechos humanos de las mujeres, Unidad 1, lectura 2, Derechos del Hombre. [En línea] [Consulta: 7 de julio de 2016]. Disponible en: https:// aulainteramericana.ac.cr/mod/folder/view.php?id=3902 
lenguaje sexista y no inclusivo, establece en su artículo 2 la igualdad ante la ley y la no discriminación, y determina en su artículo 7 la protección a la maternidad y a la infancia.

El mismo año y bajo una terminología más adecuada, igualitaria e inclusiva, producto de la lucha de la Comisión de la Condición Jurídica de la Mujer ${ }^{5}$, la Asamblea General de las Naciones Unidas, mediante la Resolución 217 A (III) del 10 de diciembre de 1948, emitió la Declaración Universal de los Derechos Humanos, consagrando en su artículo 1 que todos los seres humanos nacen libres e iguales en dignidad y derechos, y determinando en su artículo 2 que toda persona tiene los derechos y libertades proclamados sin distinción alguna de raza, color, sexo, idioma, religión, opinión política o de cualquier otra índole, origen nacional o social, posición económica, nacimiento o cualquier otra condición.

Posteriormente, la Asamblea General de las Naciones Unidas adoptó el primer documento sobre el estatus de la mujer, en la Convención sobre los Derechos Políticos de la Mujer, el 31 de marzo de 1953; luego se expidió la Convención sobre la Nacionalidad de la Mujer Casada, aprobada el 20 de febrero de 1957 y que entró en vigor el 11 de agosto de 1958.

El 16 de diciembre de 1966, la Asamblea General de la onU aprobó el Pacto Internacional de Derechos Civiles y Políticos, que entró en vigor el 23 de marzo de 1976, destacándose el artículo 2.1, que consagra la no discriminación en el disfrute de los derechos; el artículo 3, que determina la igualdad de hombres y mujeres en el goce de los derechos; el artículo 4.1, que establece la no discriminación al determinar limitaciones a los derechos en estados de excepción; el artículo 6.5, que instituye la no aplicación de la pena de muerte a mujeres en cinta; el artículo 23.4, que implanta la igualdad de los esposos en el matrimonio, y el artículo 26, que garantiza la igualdad ante la ley y la no discriminación.

En la misma fecha, la Asamblea General de la ONU aprobó el Pacto Internacional de Derechos Económicos, Sociales y Culturales, que entró en vigor el 3 de enero de 1976, destacándose el artículo 2.2, que estipula la no discriminación en el disfrute de los derechos; el artículo 3, que determina la igualdad de mujeres y hombres en el goce de los derechos; el artículo 7, que garantiza la no discriminación de la mujer en cuanto a condiciones satisfactorias y equitativas de trabajo, y el artículo 10.2, que determina la especial protección a las madres.

5 La Comisión de la Condición Jurídica y Social de la Mujer es el principal órgano internacional intergubernamental dedicado exclusivamente a la promoción de la igualdad de género y el empoderamiento de la mujer. Se trata de una comisión orgánica dependiente del Consejo Económico y Social, creado en virtud de la Resolución 11 (II) del Consejo, de 21 de junio de 1946. 
Otro instrumento internacional de protección es la Convención Americana sobre Derechos Humanos adoptada en San José de Costa Rica el 22 de noviembre de 1969, en la Conferencia Especializada Interamericana sobre Derechos Humanos. Entró en vigor el 18 de julio de 1978, destacándose el artículo 1.1, que determina la no discriminación; el artículo 17.4, que garantiza la igualdad de derechos de los cónyuges; el artículo 24, que consagra la igualdad ante la ley, y el artículo 27.1.-2, que también refiere la prohibición de discriminación por disposiciones de suspensión de ciertos derechos.

Refiriéndonos a los instrumentos internacionales de protección específica más importantes, se tiene la Convención sobre la Eliminación de Todas las Formas de Discriminación contra la Mujer, conocida por su acrónimo en inglés como CEDAW, aprobada el 18 de diciembre de 1979 por la Asamblea General de las onv, que reúne en un único instrumento legal internacional de derechos humanos las disposiciones de instrumentos anteriores de la onU relativas a la discriminación contra la mujer, por lo que se dice que es la Carta Internacional de los Derechos Humanos de las Mujeres ${ }^{6}$. Con la finalidad de examinar los progresos realizados por los Estados parte, el artículo 21 de la CEDAW establece el Comité para la Eliminación de la Discriminación contra la Mujer, como órgano que puede realizar sugerencias y recomendaciones de carácter general. De las veinticinco recomendaciones que ha emitido dicho Comité hasta hoy, cuatro se relacionan con los derechos políticos de las mujeres directamente, y señalan cuanto sigue.

La Recomendación General n. ${ }^{\circ} 5$ determina que "los Estados Partes hagan mayor uso de medidas especiales de carácter temporal como la acción positiva, el trato preferencial o los sistemas de cupos para que la mujer se integre en la educación, la economía, la política y el empleo".

La Recomendación General n. ${ }^{\circ} 21$ señala que "el Comité desea subrayar que las disposiciones de la Recomendación General n. ${ }^{\circ} 19$ (11. ${ }^{\circ}$ período de sesiones), relativa a la violencia contra la mujer, son de gran importancia para que la mujer pueda disfrutar de sus derechos y libertades en condiciones de igualdad. Se insta a los Estados Partes a aplicar esta Recomendación general a fin de que, en la vida pública y la vida familiar, las mujeres no sean objeto de violencia por razón de su sexo, lo que las priva de manera grave de sus derechos y libertades individuales" (resaltado propio).

La Recomendación General n. ${ }^{\circ} 23$ precisa que "las implicaciones para los Estados al aplicar el artículo 7 de la CEDAW abarcan medidas en todas

$6 \quad$ El Estado Plurinacional de Bolivia firmó el 30 de mayo de 1980 la Convención sobre la Eliminación de Todas las Formas de Discriminación contra la Mujer, ratificada el 8 de junio de 1990 (fecha del depósito) mediante la Ley n. ${ }^{\circ} 1100$ promulgada el 15 de septiembre de 1989. De la misma forma, Bolivia firmó el Protocolo de la Convención sobre la Eliminación de Todas las Formas de Discriminación contra la Mujer el 10 de diciembre 1999, ratificada el 27 de septiembre 2000 (fecha de depósito) mediante la Ley n. ${ }^{\circ} 2103$ promulgada el 20 de junio de 2000. 
las esferas de la vida pública y política de un país como concepto amplio, poderes legislativo, judicial, ejecutivo y administrativo".

La Recomendación General n. ${ }^{\circ} 25$ reitera la aplicación de medidas especiales en la educación, la economía, la política y el empleo.

Los Estados parte se han comprometido a remitir informes nacionales, con una periodicidad al menos cuatrienal, sobre las medidas que han aplicado para cumplir las obligaciones contraídas tras ratificar el tratado. Además, conforme al Protocolo Facultativo Adicional de 1999, aprobado y ratificado por Bolivia mediante la Ley 2103 de 20 de junio de 2000, dicho Comité tiene la facultad de evaluar el cumplimiento efectivo de los países respecto a la Convención.

En este sentido, el informe $\mathrm{CEDAW} / \mathrm{C} / \mathrm{BOL} / \mathrm{CO} / 5-6$, referente a las observaciones finales sobre los informes periódicos quinto y sexto, establece:

El Comité insta al Estado boliviano a que:

a) Adopte medidas especiales de carácter temporal, incluidas cuotas reglamentarias, con arreglo a lo dispuesto en el artículo 4.1) de la Convención y la recomendación general núm. 25 del Comité, a fin de promover la participación de las mujeres, en particular las indígenas, en condiciones de igualdad, en los ámbitos nacional, departamental y municipal del Gobierno y en el poder judicial, así como la representación internacional del Estado Plurinacional de Bolivia, en particular en puestos de alto nivel que entrañen la adopción de decisiones, y vigile $[s i c]$ los progresos alcanzados;

b) Establezca un programa orientado a la lucha contra los estereotipos que existen con respecto a la mujer en la política[,] creando conciencia sobre la importancia de su participación libre e igualitaria, en particular de las mujeres indígenas, en la vida política;

c) Cree programas especializados de formación y orientación sobre la capacidad de liderazgo y de negociación dirigidos a las candidatas actuales y potenciales y a las mujeres que ocupan cargos públicos; y

d) Procese y castigue debidamente a los autores de actos de violencia política por razón de género ${ }^{7}$.

La Convención Interamericana para Prevenir, Sancionar y Erradicar la Violencia contra la Mujer, adoptada y abierta a la firma, ratificación y adhesión por la Asamblea General de la oEA, en su vigésimo cuarto periodo ordinario de sesiones, del 9 de junio de 1994, en Belém do Pará (Brasil), y puesta en

7 Ministerio de Justicia de Bolivia. CEDAW. Cómo enfrentar la discriminación contra la mujer en Bolivia. Observaciones al Estado Plurinacional de Bolivia-07/2015. onu Mujeres Bolivia, 2015, 14. 
vigor el 5 de marzo de 1995, se constituye en el primer instrumento legal internacional en el mundo que reconoce el derecho de las mujeres a vivir una vida libre de violencia y cataloga la violencia contra las mujeres como una violación a los derechos humanos ${ }^{8}$. Con la finalidad de examinar los progresos realizados por los Estados parte se implementa la Comisión Interamericana de Mujeres (CIM) como un organismo de la oEA establecido en 1928. Fue el primer órgano en el mundo creado expresamente con el propósito de asegurar el reconocimiento de los derechos civiles y políticos de las mujeres. La cim está constituida por 34 delegadas titulares, una por cada Estado miembro, designadas por sus respectivos gobiernos. La Convención de Belém do Pará le confiere a la ciм la atribución de examinar los informes estatales sobre prevención, erradicación y sanción de la violencia contra las mujeres; y también la de solicitar opiniones consultivas a la Comisión y la Corte Interamericanas de Derechos Humanos, lo cual es fundamental para la interpretación de la Convención ${ }^{9}$.

La Declaración y Plataforma de Acción de Beijing, aprobada en la Cuarta Conferencia Mundial sobre la Mujer en septiembre de 1995, se centra en 12 aspectos relativos a la implementación de los derechos humanos de la mujer y establece un programa para el empoderamiento de esta, incluyendo una serie de objetivos estratégicos para eliminar la discriminación contra la mujer y lograr la igualdad entre mujeres y hombres. Conlleva estrategias políticas y jurídicas a escala mundial basadas en los derechos humanos.

En el año 2000, la comunidad internacional acordó ocho objetivos de desarrollo, denominados Objetivos de Desarrollo del Milenio (ODM), que debían cumplirse para el año 2015. Uno de estos objetivos se refiere a la igualdad de género y el empoderamiento de la mujer, y otro a la reducción de la mortalidad materna.

En la Conferencia de las onu sobre el Desarrollo Sostenible "Río+20", celebrada en el Brasil en el año 2012, los países renovaron su compromiso político en favor del desarrollo sostenible, acordaron establecer una serie de objetivos de desarrollo sostenible y crearon un foro político de alto nivel sobre el desarrollo sostenible. Es importante destacar que el documento final, "El futuro que queremos", también reafirma los compromisos de los Estados de asegurar a las mujeres la igualdad de derechos, acceso y oportunidades de participación y liderazgo en la economía, la sociedad y la adopción de decisiones políticas; a la vez que contiene referencias explícitas

8 El Estado Plurinacional de Bolivia ratificó la Convención Interamericana para Prevenir, Sancionar y Erradicar la Violencia contra la Mujer (Convención de Belém do Pará) por medio de la Ley n. ${ }^{\circ} 1599$ de 18 de agosto de 1994, en la que mediante un artículo único se aprueban y ratifican los articulados y compromisos de la Convención y conforma el Mecanismo de Seguimiento de la Convención de Belém do Pará (MESECVI).

9 Cамасно. Acercándonos a los instrumentos internacionales de protección de los derechos humanos de las mujeres, cit., 82 . 
a la aceleración del cumplimiento de los compromisos que figuran en la Convención sobre la Eliminación de Todas las Formas de Discriminación contra la Mujer, la Plataforma de Acción de Beijing y la Declaración del Milenio. En el documento final también se dice que la igualdad entre los géneros y la participación efectiva de las mujeres son importantes para la adopción de medidas eficaces en todos los aspectos del desarrollo sostenible, y se pide la derogación de las leyes discriminatorias así como el acceso de las mujeres a la justicia en condiciones de igualdad ${ }^{10}$.

En el año 2015, los países del mundo coincidieron en la necesidad de contar con un financiamiento integral para el desarrollo sostenible y lograron un nuevo acuerdo mundial sobre cambio climático. El documento "Transformando nuestro mundo: la agenda 2030 para el desarrollo sostenible" fue adoptado formalmente por las y los líderes del mundo durante la Cumbre de la onu, celebrada en Nueva York del 25 al 27 de septiembre de 2015. La agenda plantea 17 objetivos con 169 metas de carácter integrado e indivisible que abarcan las esferas económica, social y ambiental. Los Objetivos de Desarrollo Sostenible (ODS) delinean la nueva estrategia que regirá los programas de desarrollo mundiales durante los próximos 15 años, abordando retos fundamentales como la pobreza, la desigualdad y la violencia contra las mujeres. Contemplan un objetivo específico para la igualdad de género y el empoderamiento de las mujeres (oDs 5), además de metas que incorporan la perspectiva de género de manera transversal en los demás objetivos. En este contexto, el objetivo 5.5 propone asegurar la participación plena y efectiva de las mujeres y la igualdad de oportunidades de liderazgo a todos los niveles decisorios en la vida política, económica y pública ${ }^{11}$.

Por razones de espacio ${ }^{12}$, finalizamos los antecedentes de la evolución de la protección de los derechos civiles y políticos de las mujeres refiriendo que

10 Oficina del Alto Comisionado de los Derechos Humanos de las Naciones Unidas. Los derechos de la mujer son derechos humanos. Nueva York y Ginebra, 2014, 14-16. [En línea] [Consulta: 21 de julio de 2016]. Disponible en: http://www.ohchr.org/Documents/Publications/ HR-PUB-14-2_SP.pdf

11 ONu. Agenda 2030 y los Objetivos de Desarrollo Sostenible, una oportunidad para América Latina y el Caribe. Julio, 2016, 14.

12 La Organización Internacional del Trabajo (оIт) fue creada después de la Primera Guerra Mundial, en 1919, como parte XIII del Tratado de Versalles, cuando se reunió la Conferencia de la Paz; esta organización adoptó una serie de convenciones para proteger a la mujer trabajadora, tales como: el Convenio n. ${ }^{\circ} 100$ sobre la igualdad de las remuneraciones, de 1951, que determina que las mujeres tienen derecho a percibir el mismo salario que los hombres cuando realizan un trabajo del mismo valor que estos; el Convenio $n .^{\circ} 111$, sobre la discriminación en el empleo y ocupación, de 1958, que determina que ninguna persona puede ser discriminada en su empleo u ocupación por motivos de raza, color, sexo, ideas políticas, creencias religiosas, condición social; el Convenio n. ${ }^{\circ} 156$, sobre los trabajadores con responsabilidades familiares, de 1981 , que determina que todas las trabajadoras y trabajadores con responsabilidades familiares tienen derecho a protección especial y a no ser discriminados en el empleo y ocupación por esta condición; el Convenio n. ${ }^{\circ}$ 183, sobre la protección de la maternidad, del año 2000, que revisa 
en la vi Conferencia de Estados Parte de la "Convención Interamericana para prevenir, sancionar y erradicar la violencia contra las mujeres", celebrada en Lima en octubre de 2015, se adoptó la Declaración sobre la Violencia y el Acoso Políticos contra las Mujeres, como primer acuerdo regional íntegro sobre esta problemática, en el marco de la Convención de Belém do Pará. Esta Declaración es de vital importancia porque representa el consenso regional actual de los Estados parte de la Convención sobre el diagnóstico, la definición del problema y las acciones que se pueden tomar para prevenir, sancionar y erradicar la violencia política contra las mujeres.

\section{LA SITUACIÓN DE JURE DE LA PARTICIPACIÓN POLÍTICA DE LAS MUJERES EN EL CONTEXTO BOLIVIANO}

La Constitución boliviana contiene un listado importante de artículos sobre participación y ciudadanía para las mujeres. Así, el artículo 11 señala: "La República de Bolivia adopta para su gobierno la forma democrática participativa, representativa y comunitaria, con equivalencia de condiciones entre hombres y mujeres". Por otra parte, el artículo 26 dispone: “Todas las ciudadanas y los ciudadanos tienen el derecho a participar libremente en la formación, ejercicio y control del poder político, directamente o por medio de sus representantes, de manera individual o colectiva. La participación será equitativa y en igualdad de condiciones entre hombres y mujeres".

Los artículos 172.22 y 278.II explicitan la participación en equivalencia e igualdad de condiciones entre hombres y mujeres, en la conformación del gabinete ministerial y las asambleas departamentales, lo que abre el camino a la paridad y alternancia de género. El Decreto Supremo 29894, de 7 de febrero de 2009, establece la estructura organizativa del Órgano Ejecutivo del Estado Plurinacional, y en su artículo 14.r.12 se incorpora la participación de las mujeres como servidoras en todas las instituciones estatales.

La Ley 4021 de Régimen Electoral Transitorio, de 14 de abril de 2009; la Ley 018 del Órgano Electoral, de 16 de junio de 2010, y la Ley 026, de Régimen Electoral, de 30 de junio de 2010, incorporan los principios constitucionales de inclusión, no discriminación, igualdad de oportunidades, equidad de género, equivalencia, paridad y alternancia en la participación política de las mujeres.

El artículo 2, literales e y h de la Ley del Régimen Electoral, ordena la igualdad y equivalencia de observancia obligatoria entre los principios que

y actualiza el Convenio 103 para la protección de la maternidad, con el objetivo de seguir promoviendo la igualdad de todas las mujeres integrantes de la fuerza de trabajo así como la salud y la seguridad de la madre y el niño. En el artículo I se estipula que el término "mujer" se aplica a toda persona de sexo femenino, sin ninguna discriminación, y el término "hijo" a todo hijo, sin ninguna discriminación. Cfr. oIт. Promoviendo la igualdad de género, Convenios de la ort y los derechos laborales de las mujeres. Ginebra, 2006. 
rigen el ejercicio de la democracia intercultural. Además, reconoce en el artículo 4 la equivalencia de condiciones entre mujeres y hombres para el ejercicio de los derechos políticos. El artículo 11 garantiza la equivalencia de condiciones, y obliga a las autoridades a garantizar y cumplir con la equidad de género y la igualdad de oportunidades entre mujeres y hombres, con base en los criterios de alternancia y paridad. Asimismo, establece que en las listas de candidaturas a senadoras y senadores, diputadas y diputados, asambleístas departamentales y regionales, concejalas y concejales municipales y otras autoridades electivas, se garantizará que exista una candidata titular mujer y, a continuación, un candidato titular hombre; un candidato suplente hombre y, a continuación, una candidata suplente mujer, de manera sucesiva.

En casos de elección de una sola candidatura en una circunscripción, como son los de diputadas y diputados uninominales y asambleístas departamentales por territorio, la paridad y alternancia de género se expresará en titulares y suplentes, y en el total de las circunscripciones titulares por lo menos el $50 \%$ de las candidaturas titulares corresponderán a mujeres. El artículo hace mención específica a que las listas de las candidatas y candidatos "de las naciones y pueblos indígena originario campesinos" [sic] respetarán los mismos criterios.

El artículo 13 de la Ley 027 del Tribunal Constitucional Plurinacional, de 6 de julio de 2010, señala que el contralor constitucional debe estar conformado por siete magistradas y magistrados titulares, y siete magistradas y magistrados suplentes, y que al menos dos magistradas y magistrados provendrán del sistema indígena originario campesino. Un avance en el Órgano Judicial es la designación de una profesional indígena originaria como magistrada, ahora como parte del Consejo de la Magistratura.

La Ley 018 del Órgano Electoral Plurinacional determina la participación de las mujeres en el referido órgano en todos sus niveles, teniendo como principio el de la equivalencia, equidad de género e igualdad de oportunidades entre hombres y mujeres, para asegurar el derecho de votar y ser elegidas en elecciones públicas. Según su artículo 12, en el Tribunal Supremo Electoral se garantiza la composición de siete miembros, de los cuales por lo menos tres serán mujeres. El artículo 33 determina que en los tribunales electorales departamentales la composición es de cinco miembros, de los cuales dos serán mujeres. Estos tribunales tienen la obligación de verificar en todo el proceso electoral el estricto cumplimiento del principio de equivalencia, garantizando la paridad y alternabilidad entre hombres y mujeres según lo establecido en los artículos 23.6, 24.16 y 38.9 de la referida ley.

La Ley 031, Marco de Autonomías y Descentralización “Andrés Ibáñez", de 19 de julio de 2010, en su artículo 5.11 garantiza el ejercicio pleno de las libertades y los derechos de mujeres y hombres para alcanzar el logro de justicia social e igualdad de oportunidades. Las bases para el régimen de autonomías se asientan en los artículos 7.II.8 y 12.I, que establecen los principios de equidad e 
igualdad de oportunidades. Asimismo, la equidad y la igualdad de oportunidades son aspectos que deben ser tomados en cuenta en las diferentes formas o tipos de autonomías, según ordenan los artículos 30.1 y 32.I, donde los estatutos y las cartas orgánicas deben ser redactadas bajo un régimen de igualdad de género, generacional. El artículo 93.Iv.1 establece que los planes de desarrollo municipal se deben enfocar en el desarrollo humano, con equidad de género.

El artículo 238, literal p, de la Ley 026, de Régimen Electoral, reconoce el acoso político como delito electoral, estableciendo que la persona que hostigue a una candidata o candidato con el objeto de obtener contra su voluntad la renuncia a su postulación o a su cargo será sancionada con pena privativa de libertad de dos a cinco años. Si bien esta previsión normativa es importante para abordar los frecuentes casos de acoso a mujeres políticas, no protege a mujeres que son electas y tampoco explicita otras formas de violencia política.

Finalizamos este punto refiriéndonos a la Ley 243, de 28 de mayo del 2012, contra el Acoso y Violencia Política hacia las Mujeres, que por su importancia será desarrollada infra en nuestro trabajo.

\section{LA SITUACIÓN DE FACTO DE LA PARTICIPACIÓN POLÍTICA \\ DE LAS MUJERES EN EL CONTEXTO BOLIVIANO}

\subsection{La participación política de las mujeres a nivel nacional ${ }^{13}$}

La distribución de escaños resultante de las elecciones de octubre de 2014 muestra un porcentaje del 44,44\% (16 escaños) de mujeres en el Senado y de 50,7\% (66 escaños) en la Cámara de Diputados y Diputadas. Los 166 escaños de la Asamblea Legislativa Plurinacional para la Gestión 2015-2019 están distribuidos entre un 49,3\% (82 escaños) de mujeres y un 50,6\% (84 escaños) de hombres. Según el Mapa Mujeres en la Política 2014, elaborado por la Unión Interparlamentaria y ONU Mujeres ${ }^{14}$, estas cifras colocan a Bolivia en el tercer lugar del mundo en participación política de las mujeres en cargos parlamentarios, tras Ruanda y Andorra, ascendiendo 48 puestos (entre 150 países medidos) respecto de su situación a 1. ${ }^{\circ}$ de enero de 2014 y superando, incluso, la media regional de los países nórdicos $(42,1 \%)$.

13 En cinco países se ha incorporado la paridad a nivel estatal: Bolivia, Ecuador y México en sus constituciones, y Costa Rica y Nicaragua en su legislación secundaria.

14 El mapa puede ser consultado en: http://www.ipu.org/pdf/publications/wmnmap14_ sp.pdf 
COMPOSICIÓN POR SEXO DE LA ASAMBLEA LEGISLATIVA PLURINACIONAL ELECCIONES 2009 Y 2014

\begin{tabular}{|l|c|c|c|c|c|c|}
\hline \multirow{2}{*}{ CARGO } & \multicolumn{2}{|c|}{ HOMBRES } & \multicolumn{2}{c|}{ MujERES } & \multicolumn{2}{c|}{ TotAL } \\
\hline Año & 2009 & 2014 & 2009 & 2014 & 2009 & 2014 \\
\hline Total Senadores/senadoras & 19 & 20 & 17 & 16 & 36 & 36 \\
\hline Porcentaje & $53 \%$ & $55,6 \%$ & $47 \%$ & $44,4 \%$ & $100 \%$ & $100 \%$ \\
\hline Total Diputados/diputadas & 100 & 64 & 30 & 66 & 130 & 130 \\
\hline Porcentaje & $77 \%$ & $49,2 \%$ & $23 \%$ & $50,7 \%$ & $100 \%$ & $100 \%$ \\
\hline Total Asamblea Legislativa & 119 & 84 & 47 & 82 & 166 & 166 \\
\hline Porcentaje & $71,68 \%$ & $50,6 \%$ & $28,31 \%$ & $49,3 \%$ & $100 \%$ & $100 \%$ \\
\hline
\end{tabular}

Fuente: Tribunal Supremo Electoral, 2010-2014.

Según datos del Ministerio de Comunicación de Bolivia, en el Órgano Ejecutivo existen actualmente 21 carteras ministeriales, de las cuales 15 están dirigidas por hombres y 6 por mujeres ${ }^{15}$, es decir el $72 \%$ y el $28 \%$ respectivamente. No obstante, al inicio de la anterior legislatura (2010-2014) la presencia de mujeres en el Gabinete Ministerial era paritaria, si bien sucesivas reformas implicaron una reducción muy significativa en el número de mujeres ministras.

\section{PRESENCIA DE MUJERES EN GABINETES MINISTERIALES \\ EN EL PERIODO 2006-2015}

\begin{tabular}{|c|c|c|c|}
\hline Gestión GUBERNAMENTAL & $\begin{array}{c}\text { N. }{ }^{\circ} \text { DE CARTERAS } \\
\text { MINISTERIALES }\end{array}$ & N. ${ }^{\circ}$ DE MUJERES & \% DE MUJERES \\
\hline $2006-2009$ & 20 & 4 & $20 \%$ \\
\hline $2010-2011$ & 20 & 10 & $50 \%$ \\
$2012-2013$ & 20 & 7 & $35 \%$ \\
$2013-2014$ & 21 & 7 & $33 \%$ \\
\hline 2015 (febrero-agosto) & 21 & 6 & $28 \%$ \\
\hline
\end{tabular}

Fuente: Coordinadora de la Mujer y Ministerio de Comunicación, 2006-2015.

Según la Coordinadora de la Mujer, en el Órgano Judicial ${ }^{16}$, de 28 autoridades elegidas en las elecciones judiciales de 2011, 16 son hombres (57\%)

15 Ocupan actualmente las carteras de Desarrollo Productivo y Economía Plural, Justicia, Salud, Medio Ambiente y Agua, Transparencia Institucional y Lucha contra la Corrupción y Comunicación.

16 El Órgano Judicial de Bolivia está compuesto por el Tribunal Supremo de Justicia, el Tribunal Constitucional Plurinacional, el Tribunal Agroambiental y el Consejo de la Magistratura. 
y 12 son mujeres (43\%). El mayor porcentaje, en 2011, se concentraba en el Tribunal Constitucional Plurinacional (presidido por un hombre), con un 57\%; le seguía el Tribunal Agroambiental (presidido por un hombre), con un 43\%; muy cerca, el Consejo de la Magistratura, con un 40\% (presidido por una mujer) y, finalmente, con un 33\%, el Tribunal Supremo de Justicia (presidido por un hombre ${ }^{17}$ ) ${ }^{18}$. Para el año 2015 estos datos habían tenido muy pocas variaciones y tan solo el Tribunal Constitucional Plurinacional había modificado sus porcentajes por cambios sucedidos en el período.

PORCENTAJE DE MUJERES EN EL ÓRGANO JUDICIAL,

ELECCIÓN 2011 Y COMPOSICIÓN EN 2015

\begin{tabular}{|l|c|c|}
\hline \multicolumn{1}{|c|}{ ÓRgano Judicial } & $\begin{array}{c}\text { \% DE Mujeres } \\
\text { (ElECTAS EN 2011) }\end{array}$ & $\begin{array}{c}\% \text { DE MuJERES } \\
\text { (OCTUBRE 2015) }\end{array}$ \\
\hline Tribunal Agroambiental (7 mag.) & $42,8 \%$ (3 mujeres) & $42,8 \%$ (3 mujeres) \\
\hline Consejo de la Magistratura (5 mag.) & $40 \%$ (2 mujeres) & $40 \%$ (2 mujeres) \\
\hline Tribunal Constitucional Plurinacional (7 mag.) & $42,8 \%$ (3 mujeres) & $28,5 \%$ (2 mujeres) \\
\hline Tribunal Supremo de Justicia (9 mag.) & $33 \%$ (3 mujeres) & $33 \%$ (3 mujeres) \\
\hline
\end{tabular}

Fuente: Coordinadora de la Mujer, 2013, y web institucionales, 2015.

En el Tribunal Supremo Electoral, la distribución actual tras el último proceso de elección parlamentaria de sus miembros es de 3 hombres (43\%) y 4 mujeres (57\%), estando la presidencia en cabeza de una mujer ${ }^{19}$. En este último caso, una Reglamentación para la Elección de Vocales emitida al efecto por la Asamblea Legislativa Plurinacional ${ }^{20}$ indica, en su artículo 11, una serie de condiciones habilitantes para garantizar la paridad cuantitativa entre los sexos.

17 A mediados de octubre de 2015 el presidente del Tsu presentó su dimisión y a principios de noviembre de 2015 el organismo eligió a un hombre como presidente.

18 El Tribunal Supremo de Justicia alberga al Comité de Género del Órgano Judicial de Bolivia, en el que participan la totalidad de magistradas de los tres tribunales y del Consejo de la Magistratura. El Comité fue creado en 2014.

19 Las y los magistrados del Tribunal Supremo Electoral fueron elegidos en las elecciones judiciales de 2011; sin embargo, en 2015 se produjo su dimisión completa y se desarrolló, de la mano de la Asamblea Legislativa Plurinacional, un proceso selectivo a partir de la postulación libre de ciudadanos y ciudadanas supuestamente con base en criterios meritocráticos.

20 Puede consultarse en línea en: http://www.diputados.bo/index.php/noticias/2178reglamento-para-la-designacion-de-vocales-del-tribunal-supremo-electoral 
PORCENTAJE DE MUJERES EN EL ÓRGANO ELECTORAL, ELECCIÓN 2011 Y COMPOSICIÓN EN 2015

\begin{tabular}{|c|c|c|}
\hline Órgano Electoral & $\%$ DE HOMBres & $\%$ DE MUJERES \\
\hline (Composición 2011) Tribunal Supremo Electoral & $57 \%$ (4 hombres) & $43 \%$ (3 mujeres) \\
\hline (Composición 2015) Tribunal Supremo Electoral & $43 \%$ (3 hombres) & $57 \%$ (4 mujeres) \\
\hline
\end{tabular}

Fuente: Tribunal Supremo Electoral, 2010-2015.

\subsection{La participación política de las mujeres a nivel departamental}

Los datos referidos a los gobiernos autónomos departamentales muestran la misma tendencia que los municipales; si bien tanto en las elecciones subnacionales de 2010 como en las de 2015 ninguna mujer fue elegida gobernadora $^{21}$, la presencia de mujeres en las asambleas departamentales aumentó sustantivamente debido a la aplicación de la nueva legislación y a la vigilancia del movimiento de mujeres.

COMPOSICIÓN POR SEXO DE LAS ASAMBLEAS DEPARTAMENTALES ELECCIONES SUBNACIONALES DE 2010 Y 2015

\begin{tabular}{|l|c|c|c|c|c|c|}
\hline \multirow{2}{*}{ CARgo } & \multicolumn{2}{c|}{ Hombres } & \multicolumn{2}{c|}{ Mujeres } & \multicolumn{2}{c|}{ Total } \\
\hline Año & 2010 & 2015 & 2010 & 2015 & 2010 & 2015 \\
\hline Asambleístas departamentales & 191 & 149 & 76 & 123 & 267 & 272 \\
\hline Porcentaje & $71,53 \%$ & $54,77 \%$ & $28,47 \%$ & $45,22 \%$ & $100 \%$ & $100 \%$ \\
\hline
\end{tabular}

Fuente: Tribunal Supremo Electoral, 2010-2015; Coordinadora de la Mujer, 2015, y ACoвoL, 2015.

\subsection{La participación política de las mujeres a nivel municipal}

Datos de la Asociación de Concejalas de Bolivia (ACOBOL), de la Coordinadora de la Mujer y del Tribunal Supremo Electoral referidos a las elecciones subnacionales de 2015 indican, en efecto, un claro predominio masculino en las alcaldías municipales aunque, obviamente, al aplicarse la legislación, y gracias a la vigilancia del movimiento de mujeres, el porcentaje de concejalas ha aumentado hasta llegar a la paridad cuantitativa entre los sexos.

21 En las elecciones subnacionales de 2015 tan solo tres mujeres fueron candidatas. 
COMPOSICIÓN POR SEXO DE LOS CONCEJOS MUNICIPALES

ELECCIONES SUBNACIONALES DE 2010 Y 2015[22]

\begin{tabular}{|l|c|c|c|c|c|c|}
\hline \multicolumn{1}{|c|}{ CARGO } & \multicolumn{2}{c|}{ Hombres } & \multicolumn{2}{c|}{ Mujeres } & \multicolumn{2}{c|}{ TOtAL } \\
\hline Año & 2010 & 2015 & 2010 & 2015 & 2010 & 2015 \\
\hline $\begin{array}{l}\text { Concejales/ } \\
\text { concejalas }\end{array}$ & 1.045 & 997 & 786 & 1.028 & 1.831 & 2.025 \\
\hline Porcentaje & $57,07 \%$ & $49,23 \%$ & $42,93 \%$ & $50,77 \%$ & $100 \%$ & $100 \%$ \\
\hline
\end{tabular}

Fuente: Tribunal Supremo Electoral, 2010-2015; Coordinadora de la Mujer, 2015, y ACOBOL, 2015.

Según onu Mujeres, tras las elecciones generales de 2014 y subnacionales de 2015, Bolivia es el único país en América Latina que ha logrado resultados de paridad, a través de las normas paritarias que ha adoptado en su sistema legislativo (con el 51\% de mujeres en la Asamblea Legislativa Plurinacional, el $45 \%$ en las asambleas departamentales y el $51 \%$ en los concejos municipales) ${ }^{23}$.

\section{ACOSO Y VIOLENCIA POLÍTICA CONTRA LAS MUJERES}

No obstante los progresos descritos supra, los principales problemas relacionados con la vulneración de los derechos políticos de las mujeres surgen en los ámbitos departamental y municipal. En efecto, según onU Mujeres ${ }^{24}$, "[e]n Bolivia no existe ninguna mujer gobernadora y solo 27 de los 339 municipios (cerca del 8\%) tienen una Alcaldesa" 25.

De manera concordante con estos datos, se ha generado un incremento sistemático de actos de acoso y violencia política, principalmente en municipios. Es así que desde el inicio de la gestión de las concejalas electas en el año 2010, fueron recepcionadas un total de 272 denuncias, registrando un $11 \%$ anterior a la promulgación de la Ley 243. Los mayores índices de denuncia se suscitaron desde la gestión 2012, con un 89\%; los departamentos donde se produjeron más hechos de violencia son Chuquisaca, La Paz y Potosí, entre un total de 170 municipios que representan el 50\% del total. La mayor cantidad de hechos de acoso y violencia política se dieron en la gestión

22 En 2010 el total de municipios existentes en el país era de 336, mientras que en 2015 era de 339 .

23 ONU Mujeres América Latina y el Caribe. Datos de ACOBOL sobre los resultados de las elecciones de 2015. [En línea]. [Consulta: 27 de julio de 2016]. Disponible en: http://lac.unwomen.org/es/noticias-y-eventos/articulos/2016/04/bolivia-paridad

24 ONU Mujeres es la organización de las Naciones Unidas dedicada a promover la igualdad de género y el empoderamiento de las mujeres. Como defensora mundial de mujeres y niñas, ONU Mujeres fue establecida para acelerar el progreso que conducirá a mejorar las condiciones de vida de las mujeres y para responder a las necesidades que enfrentan en el mundo.

25 ONu Mujeres América Latina y el Caribe, cit. 
2013, considerando que las concejalas se encontraban a mitad de gestión; en primer término por presión para que renunciaran al cargo de concejala, y luego por una errónea e ilegal interpretación sobre la gestión compartida, según refleja el siguiente cuadro ${ }^{26}$.

DETALLE DE CASOS DE ACOSO Y/O VIOLENCIA POLÍTICA EN MUNICIPIOS

\begin{tabular}{|c|c|c|c|c|c|c|c|}
\hline DEPARTAMENTO & $\begin{array}{c}\text { N. }{ }^{\circ} \text { DE } \\
\text { MUNICIPIOS }\end{array}$ & 2010 & 2011 & 2012 & 2013 & $\begin{array}{c}\text { 2014 (HASTA } \\
\text { SEPTIEMBRE) }\end{array}$ & $\begin{array}{c}\text { TOTAL } \\
\text { DE CASOS } \\
2010-2014\end{array}$ \\
\hline Pando & 14 & 0 & 2 & 3 & 29 & 1 & 35 \\
\hline Cochabamba & 15 & 1 & 0 & 3 & 9 & 3 & 16 \\
\hline Tarija & 5 & 0 & 1 & 0 & 1 & 3 & 5 \\
\hline Potosí & 40 & 4 & 4 & 17 & 16 & 7 & 48 \\
\hline Santa Cruz & 35 & 5 & 0 & 6 & 14 & 12 & 37 \\
\hline Chuquisaca & 13 & 4 & 1 & 8 & 43 & 8 & 64 \\
\hline Oruro & 4 & 4 & 0 & 0 & 3 & 1 & 8 \\
\hline La Paz & 39 & 5 & 0 & 3 & 35 & 11 & 54 \\
\hline Beni & 5 & 0 & 0 & 0 & 4 & 1 & 5 \\
\hline Total & 170 & 23 & 8 & 40 & 154 & 47 & 272 \\
\hline
\end{tabular}

Fuente: АСОвоL, 2014.

Como acertadamente refiere ACOBOL, las mujeres que ocupan cargos de decisión en el nivel municipal están siendo afectadas por actos sistemáticos de acoso y violencia que vulneran sus derechos políticos. Otras autoridades municipales y sociales están realizando acciones con el afán de restringir o destituir del cargo a las mujeres, recurriendo indebidamente a actos de acoso y violencia política; prueba de ello son los datos de las gestiones 2010 a 2014, que reflejan que 200 concejalas no iniciaron ninguna acción legal debido, entre otras causas, a desconocimiento de las instituciones que atienden estos casos, a miedo a la reacción de sus hostigadores y a miedo a la represión de las organizaciones sociales.

De un total de 272 casos que se produjeron, solo en 72 se presentaron denuncias ante instancias judiciales, el Órgano Electoral Plurinacional y el Concejo Municipal. Como resultado de estas acciones, en la gestión 2013

26 Cfr. Asociación de Concejalas de Bolivia - AcoBol. Boletín de la Asociación de Concejalas de Bolivia - ACOBOL, n. ${ }^{\circ}$ 7, diciembre de 2014. [En línea]. [Consulta: 27 de julio de 2016]. Disponible en: http://www.autonomias.gob.bo/portal3/images/stories/minifp/2015/Archivos_PDF/ genero_7_boletin\%20_concejala.pdf 
no se tenía registrado ningún caso resuelto por acoso y violencia política. Sin embargo, hasta septiembre de 2014 se resolvieron 13 casos (18\%) por la vía administrativa y constitucional, siendo de lamentar que a la fecha se tienen 0 casos resueltos por la vía penal. El retardo de justicia y la falta de sensibilización de los operadores de justicia causan que de 42 casos denunciados a las instancias judiciales solo 4 hayan sido resueltos por acciones de amparo constitucional.

De 249 testimonios sobre diferentes casos denunciados de acoso y violencia política, con un promedio de 28 casos por año (ACOBOL, 2011, 2), los casos más denunciados fueron por: (i) presión para la renuncia de concejalas, (ii) actos de violencia sexual, física y psicológica así como exceso de autoridad contra concejalas; (iii) impedimento en el ejercicio de sus funciones y ocupación ilegal de la concejalía; (iv) congelamiento ilegal de salarios; (v) discriminación, y (vi) difamación y calumnia.

Del total de casos denunciados, el $40 \%$ fueron derivados a instancias públicas de gobierno, quedando todos en la impunidad. El 32\% no obtuvo ninguna respuesta y en relación con el 7,6\% las instituciones se excusaron indicando no tener competencia al respecto ${ }^{27}$.

\subsection{Juana Quispe Apaza, uno de los casos más extremos de acoso y violencia política ${ }^{28}$}

Un caso extremo, muy bien reflejado por Roxana Vásquez, es el de Juana Quispe Apaza, quien fue concejala titular del Municipio de Ancoraimes, provincia Omasuyos, en representación de la agrupación ciudadana FSUTCLP-TK.

[E]lla tuvo problemas desde el inicio para el ejercicio de su cargo con los integrantes del Concejo Municipal y con el Alcalde de dicho municipio. Además de

27 Cfr. AсовоL. [En línea]. [Consulta 27 julio 2016]. Disponible en: http://www.acobol.org. bo/site/index.php/numero-de-casos-de-acoso-y-violencia-politica-resueltos-por-vias-de-denuncia

28 En el contexto latinoamericano existen también casos extremos de acoso y violencia política, como el acontecido en México donde la precandidata por el PRD, Aída Nava, fue encontrada decapitada en las inmediaciones del poblado de Tecoanapa, un día después de haber sido secuestrada durante un acto político; o el caso de la precandidata a la diputación federal por el Pan en Morelos, Gabriela Pérez Cano, que sufrió un ataque en su domicilio, durante el cual los agresores se llevaron objetos de valor y documentos importantes, además de dejar un mensaje que decía: "Abandona la candidatura" y agredir a su hijo de 14 años; o el caso de Gisela Mota, alcaldesa de Temixco, asesinada un día después de asumir el cargo. Tribunal Electoral del Poder Judicial de la Federación. Protocolo para atender la violencia política contra las mujeres. México: Coordinación de Comunicación Social, 2016, 13. [En línea]. [Consulta: 3 de agosto de 2016]. Disponible en: http://www.fepade.gob.mx/actividades_ins/2016/marzo/ProtocoloViolencia_140316.pdf No deja de ser menos importante recordar que cada 25 de noviembre se recuerda el día contra la violencia hacia las mujeres, en conmemoración de la muerte violenta de las hermanas Mirabal, activistas políticas de República Dominicana que fueron asesinadas en 1960 por su oposición al régimen de Rafael Trujillo. 
tener que enfrentar de manera constante y sistemática violencia física, verbal y amenazas de ciertos actores y sectores institucionales, políticos y sociales. En una determinación justa y legal, el Órgano Electoral Departamental reconoce como válida, en todos sus sentidos y contenidos, la demanda de amparo constitucional interpuesta por Juana en el año 2010, restituyéndosele todos sus derechos y garantías como concejala titular. Sin embargo, el Gobierno Autónomo Municipal de Ancoraimes no la repone en el cargo. Ella continúa su lucha y presenta una segunda acción de amparo, la misma que es resuelta a su favor a finales de 2011. En esta oportunidad, sí se cumple con restituirla en el cargo. Sin embargo, acabaron con su vida unas semanas después. Como antecedente, cabe señalar que las denuncias realizadas por Juana por atentados contra su seguridad y su vida por más de dos años no fueron escuchadas. Tuvo que enfrentarse a procesos administrativos y judiciales interminables y a la violación permanente de sus derechos humanos y libertades fundamentales. Las audiencias convocadas por la autoridad competente se convirtieron en verdaderos cercos de violencia verbal y física y de amenazas contra ella. Se tiene constancia de que el día de su asesinato fue una vez más a la fiscalía a denunciar que estaba siendo perseguida y a pedir garantías para su vida. Impedirle ejercer su función pública como concejala y retener su salario por casi dos años, fueron los "problemas" menores que debió enfrentar, si los comparamos con las agresiones físicas de las que fue objeto como tirarle cemento a los ojos, insultarla públicamente y amenazarla de muerte hasta que se halló su cuerpo sin vida. En la revisión documental se pudo constatar que lo señalado por Juana Quispe Apaza desde el 2010 hasta la fecha de su asesinato era cierto, tal como lo corroboraron algunas organizaciones sociales, autoridades e incluso la policía del Municipio. También se cuenta con información relativa a que autoridades institucionales, comunales y representantes de ciertas organizaciones sociales no sólo le negaron el derecho a ejercer sus funciones sino que la juzgaron y sentenciaron sin prueba alguna. A más de cuatro años de su asesinato, el caso si bien ha sido judicializado, se encuentra paralizado y las personas imputadas como autoras de su asesinato se encuentran en libertad. Esta situación trae como consecuencia que los autores intelectuales y materiales continúen en la impunidad ${ }^{29}$.

\section{LA PRIMERA LEY ESPECÍFICA SOBRE ACOSO Y VIOLENCIA POLÍTICA DE AMÉRICA LATINA Y SUS CLAROSCUROS}

La Ley 243 de 28 de mayo de 2012, denominada Ley contra el Acoso y Violencia Política hacia las Mujeres ${ }^{30}$, no solo constituye la primera ley

29 Vásquez, R. El acoso y la violencia política hacia las mujeres en Bolivia. Avances formales y desafios reales para la igualdad. Bolivia: AсовоL y Cooperación Alemana al Desarrollo - GIZ, 2013, 55 [En línea]. [Consulta: 3 de agosto de 2016]. Disponible en: http://www. bivica.org/upload/violencia-politica-acoso.pdf

30 Gaceta del Estado Plurinacional de Bolivia, Ley n. ${ }^{\circ} 243$ contra el Acoso y Violencia Política hacia las Mujeres, de 28 de mayo de 2012. [En línea]. [Consulta: 7 de agosto de 2016]. Disponible en: http://www.gacetaoficialdebolivia.gob.bo/index.php/normas/buscar 
específica de América Latina sobre acoso y violencia política ${ }^{31}$, sino que en esencia es reflejo de la constante lucha por los derechos políticos de las mujeres, en especial de las autoridades electas municipales representadas por АСовоL, cuyas primeras iniciativas datan del año 2000; así las cosas, se necesitaron cerca de 12 años para que se adoptara una ley formal que rigiera a nivel nacional. La ley en cita pretende establecer mecanismos de prevención, atención y sanción contra actos individuales y colectivos de acoso y/o violencia política hacia las mujeres, inspirándose no solo en la Constitución Política del Estado sino también en los tratados e instrumentos internacionales de derechos humanos ratificados por Bolivia. Tiene como finalidad eliminar actos, conductas y manifestaciones individuales y colectivas de acoso y violencia política, garantizando el ejercicio de los derechos políticos de las mujeres mediante políticas y estrategias públicas. Uno de sus aspectos más importantes es la incorporación de los actos de acoso y violencia política como nuevos tipos penales bajo las siguientes definiciones:

Artículo 148 Bis. (Acoso político contra mujeres).- Quien o quienes realicen actos de presión, persecución, hostigamiento y/o amenazas en contra de una mujer electa, designada o en el ejercicio de la función político-pública y/o de sus familiares, durante o después del proceso electoral, que impida el ejercicio de su derecho político, será sancionado con pena privativa de libertad de dos (2) a cinco (5) años.

Artículo 148 Ter. (Violencia política contra mujeres).- Quien o quienes realicen actos y/o agresiones físicas y psicológicas contra mujeres candidatas, electas, designadas o en ejercicio de la función político-pública y/o en contra de sus familiares, para acortar, suspender e impedir el ejercicio de su mandato o su función, será[n] sancionado[s] con pena privativa de libertad de tres (3) a ocho (8) años. En casos de actos o agresiones sexuales contra las mujeres candidatas, electas, designadas o en ejercicio de la función político-pública, se sancionará conforme dispone este Código Penal.

No obstante que la referida ley obtuvo la mención honrosa del Premio de Políticas del Futuro (Future Policy Award 2014) que onu Mujeres, World Future Council y la Unión Interparlamentaria conceden a las mejores leyes y políticas del mundo que persigan poner fin a la violencia ejercida contra las mujeres y las niñas, a casi tres años de su vigencia se han puesto en evidencia una serie de falencias en su contenido y aplicación. Bastará referir que el

31 Bolivia ha sido el primer país en América Latina en sancionar con una ley el acoso y la violencia política contra la mujer. Ver onu Mujeres. Guía estratégica, empoderamiento político de las mujeres. Marco para una acción estratégica, América Latina y El Caribe (2014-2017).2014, 67. [En línea]. [Consulta: 7 de agosto de 2016]. Disponible en: http://www.onumulheres.org.br/ wp-content/uploads/2016/04/EMPODERAMIENTO-POLITICO-DE-LAS-MUJERES-LAC-2014-17-UNWOMEN. pdf 
procedimiento en la vía penal al cual remite la norma para realizar denuncias no es efectivo, lo que resulta lógico si tenemos en cuenta los problemas estructurales de la crisis de la administración de justicia ordinaria ${ }^{32}$. Asimismo, el tipificar los actos de acoso o violencia política como delitos de acción pública a instancia de parte tampoco ayuda a su efectiva aplicación, ya que los operadores de justicia no realizan ningún acto investigativo sin impulso procesal de las víctimas, lo cual implica gastos económicos y contratación de abogados para intentar lograr alguna garantía mínima de justicia. En la vía constitucional, que representa otra opción para interponer la denuncia, las resoluciones de los tribunales de garantías en muchos casos no son efectivas en su cumplimiento y demoran casi un año cuando son remitidas en revisión ente el Tribunal Constitucional Plurinacional, produciéndose muchas veces un daño irreparable e irreversible a los derechos de las mujeres. En el caso de la vía administrativa, su artículo 16 establece que la víctima podrá denunciar el caso ante la misma institución a la que pertenece el agresor, agresores, agresora o agresoras, de acuerdo al procedimiento dispuesto en la normativa vigente; aspecto que encontramos incoherente ya que en la mayoría de los casos de acoso y violencia política contra autoridades municipales electas, los potenciales agresores son de la misma institución (alcalde y concejales). En este contexto, el procedimiento administrativo al cual remite la ley termina siendo el menos efectivo para garantizar los derechos políticos de las mujeres, dado que las comisiones de ética dependen de la máxima autoridad ejecutiva municipal, lo cual desnaturaliza todo sentido de imparcialidad y objetividad, máxime si tenemos en cuenta que los actos de acoso y/o violencia política, al ser considerados delitos, no pueden ser considerados también como faltas morales y/o éticas, como la normativa erróneamente establece. Todos estos aspectos, incluyendo el hecho de que la ley no establece ninguna medida de reparación, convierten a la normativa en enunciados meramente declarativos que distan mucho de alcanzar sus fines y objetivos, lo cual hizo necesario el desarrollo reglamentario correspondiente que nos permitimos analizar a continuación.

32 Según el informe anual de la Alta Comisionada de las Naciones Unidas para los Derechos Humanos, Bolivia no ha podido revertir la profunda crisis en la administración de justicia, caracterizada por problemas estructurales tales como la corrupción, los retrasos, la insuficiente cobertura de servicios judiciales en el país y el acceso limitado a la justicia por parte de los sectores más vulnerables. Estos problemas, entre otros, generan un preocupante rezago judicial y, en última instancia, impunidad. Alta Comisionada de las Naciones Unidas para los Derechos Humanos. Informe Anual Estado Plurinacional de Bolivia. 2013, párr. 45. [En línea]. [Consulta: 7 de agosto de 2016]. Disponible en: http://bolivia.ohchr.org/docs/Informe\%20Anual\%202013. pdf 


\subsection{El Decreto Supremo 2935 de 5 de octubre de 2016}

Es importante destacar que el Decreto Supremo 2935 de 5 de octubre de 2016, reglamentario de la Ley 243 de 28 de mayo de 2012, contra el Acoso y Violencia Política hacia las Mujeres, fue trabajado desde el Ministerio de Justicia en coordinación con el Ministerio de Autonomías, el Órgano Electoral Plurinacional y las organizaciones representantes de las mujeres, por lo que es también resultado de la lucha por el empoderamiento de la mujer y la construcción progresiva de una democracia paritaria plena.

La reglamentación establece en primer término un procedimiento marco para los órganos deliberativos del nivel central del Estado y las entidades territoriales autónomas. Se implementa por tanto un régimen administrativo para faltas de acoso y violencia política hacia las mujeres, para su aplicación por los órganos deliberativos, llenando el vacío normativo que no permitía la aplicación efectiva de la Ley 243 en el procesamiento de denuncias de las autoridades electas por la vía administrativa. Con la aplicación del procedimiento marco, las denuncias de acoso y violencia política en la vía administrativa para autoridades electas deberían resolverse en el término máximo de 15 días desde la recepción de la misma. El decreto en cita implementa también un mecanismo de prevención y atención inmediata para la defensa de los derechos de las mujeres en situación de acoso y/o violencia política, que será activado cuando se tenga conocimiento de un caso de acoso y/o violencia política hacia las mujeres de notoria gravedad, y/o un riesgo que ponga en peligro inminente la vida o la integridad física de la afectada y que requiera por tanto acciones inmediatas, pudiendo solicitar la intervención de la fuerza pública. La normativa determina también la implementación obligatoria de video grabaciones en las sesiones de los órganos deliberativos de las entidades territoriales autónomas, las cuales deben ser archivadas bajo responsabilidad por orden cronológico a fin de prevenir y registrar cualquier acto de acoso y violencia política.

\section{CONCLUSIÓN}

La democracia no se entiende ya sólo como un régimen político con elecciones libres de sus representantes políticos, sino como un modo de vida (PNUD 2004) que exige procesos transparentes, legítimos e inclusivos. La democracia tiene como objetivo ampliar la ciudadanía bajo los principios de inclusión, igualdad y universalidad. De ahí que la inclusión de las mujeres con plenos derechos en igualdad de condiciones y con las mismas oportunidades que los hombres sea consustancial al proceso de reforma de la cultura democrática que debe conducir también a la democracia paritaria. 
El concepto de la democracia paritaria ${ }^{33}$ trasciende lo meramente político y, por tanto, no puede limitarse a incrementar el porcentaje de representación política de las mujeres, sino que debe también impulsar un nuevo equilibrio social entre hombres y mujeres, en el que ambos asuman responsabilidades compartidas en todas las esferas de sus vidas, públicas y privadas ${ }^{34-35}$.

En ese contexto, la Corte Interamericana de Derechos Humanos ha considerado "indispensable que el Estado genere las condiciones y mecanismos óptimos para que los derechos políticos puedan ser ejercidos de forma efectiva, respetando el principio de igualdad y el principio de no discriminación" ${ }^{36}$. De manera concordante, la Comisión Interamericana de Derechos Humanos, en el caso Leopoldo López vs. Venezuela, en su análisis del contenido del artículo 23 de la Convención Americana señaló que los derechos políticos son derechos humanos de importancia fundamental dentro del sistema interamericano, que se relacionan estrechamente con un conjunto de otros derechos que hacen posible el juego democrático ${ }^{37}$.

Tomando en cuenta los principios de igualdad y no discriminación que imperan en el sistema interamericano, la Comisión observa que el involucramiento de las mujeres en todos los ámbitos de la vida política es una condición necesaria para garantizar una sociedad verdaderamente igualitaria y consolidar la democracia participativa y representativa en las Américas. La inclusión de las mujeres en la política fomenta sociedades más democráticas y la rendición de cuentas, puesto que se escuchan las voces y demandas de las mujeres, que constituyen aproximadamente la mitad de la población en las Américas y del padrón electoral. No obstante, a la par de la democracia paritaria en igualdad de condiciones, se requiere: profesionalizar la carrera política de las mujeres; mejorar su participación sustantiva en las instancias de decisión (comisiones parlamentarias, gabinetes, gubernaturas, alcaldías y tribunales), así como desarrollar cursos de capacitación y formación política para las mujeres, especialmente para las mujeres indígenas y afrodescendientes $^{38}$.

33 El concepto de democracia paritaria tuvo su primer hito en la Cumbre Europea "Mujeres en el Poder" que aprobó la Declaración de Atenas, 1992.

34 ONU Mujeres. Guía estratégica, empoderamiento político de las mujeres, cit., 51.

35 Véase onu Mujeres y Parlamento Latinoamericano y Caribeño. Norma Marco para consolidar la democracia paritaria del Parlatino.

36 Corte Interamericana de Derechos Humanos. Caso Yatama vs. Nicaragua. Excepciones Preliminares, Fondo, Reparaciones y Costas. Sentencia de 23 de junio de 2005 . Serie C, n. ${ }^{\circ} 127$, párr. 195.

37 Comisión Interamericana de Derechos Humanos, Petición internacional. Leopoldo López. Mendoza (Caso 12.668) vs. Venezuela, de 14 de diciembre de 2009, párr. 60.

38 Comisión Interamericana de Derechos Humanos. El camino hacia una democracia sustantiva: la participación política de las mujeres en las Américas. OEA/Ser.L/V/II.Doc.79, 2011, punto 51, 20. [En línea]. [Consulta: 10 de agosto de 2016]. Disponible en: https://www. cidh.oas.org/pdf\%20files/MUJERES\%20PARTICIPACION\%20POLITICA.pdf 
Se debe considerar también: las desventajas socio-económicas de las mujeres para construir una carrera política y competir en la esfera pública; el acceso limitado al financiamiento; el desconocimiento de sus derechos políticos; y, las distintas formas de violencia como obstáculo a la participación política de las mismas. Consecuentemente, uno de los mayores retos es diseñar estrategias y medidas efectivas para combatir estos obstáculos de forma que no solo exista una mayor inclusión y representación femenina en las instancias de decisión, sino una representación que garantice mayor igualdad entre los hombres y las mujeres ${ }^{39}$. Asimismo, la generación de información estadística desagregada y cualitativa es esencial para comprender la situación real de las mujeres en los países y poder así ayudar a los tomadores de decisiones públicos a adoptar las políticas públicas adecuadas que impulsen y garanticen los derechos políticos de las mujeres ${ }^{40}$.

En el caso concreto de Bolivia, la violencia en razón de género sigue siendo un problema significativo, y las mujeres jóvenes y casadas, en especial del área rural, son especialmente vulnerables a todas las formas de violencia. En el contexto de los derechos políticos, la falta de tiempo por el desempeño de tareas domésticas, el cuidado de niños pequeños, el miedo al rechazo, la falta de acceso a formación política, la objeción de la pareja, las razones económicas y la violencia política per se hacen que la democracia paritaria se reduzca a una simple participación nominal sin verdadera decisión. No obstante, es destacable la implementación del proyecto Promotoras Comunitarias "Por una Vida Libre de Violencia", donde mujeres voluntarias cumplen un rol de control social para la implementación de las normativas protectoras de mujeres en municipios y comunidades, haciendo seguimiento a la correcta inversión de los recursos económicos destinados a la lucha contra la violencia para consolidar una gestión municipal con enfoque de género ${ }^{41}$.

Consideramos que para encarar de manera efectiva este grave problema de discriminación y excusión social se necesita una región comprometida con la democracia paritaria. En esa labor, como bien refiere onU Mujeres,

El Parlamento para América Latina y el Caribe, conocido como Parlatino, ha puesto a la región en la senda de la "paridad de género". Luego de tres años de trabajo de promoción y de conocimiento experto aportado por onu Mujeres, la Asamblea, en representación de 20 países y tres territorios, redactó y aprobó en noviembre de 2015 una nueva disposición regional que orienta a los Parlamentos nacionales para alcanzar la igualdad y la paridad de género en la toma de decisiones. La resolución define un nuevo modelo de Estado inclusivo y abocado a

39 Ibíd., 41.

40 ONU Mujeres. Guía estratégica, empoderamiento político de las mujeres, cit. 55.

41 Véase Ministerio de Justicia de Bolivia. Guía de actuación de promotoras comunitarias "Por una vida libre de violencia". Bolivia: SOLIDAR Suiza-Aos, 2016, 7. 
poner fin a todas las formas de desigualdad y exclusión. Por ejemplo, exige a los países poner fin a los sesgos de género que aún persisten en la legislación, proporcionar recursos para reducir las disparidades de género y garantizar que hombres y mujeres tengan igual representación en los parlamentos, el sistema judicial y el poder ejecutivo ${ }^{42}$.

\section{REFERENCIAS}

Alta Comisionada de las Naciones Unidas para los Derechos Humanos. (2013). Informe Anual Estado Plurinacional de Bolivia.

Asociación de Concejalas de Bolivia - acobol. Boletina de la Asociación de Concejalas de Bolivia - ACOBOL, n. ${ }^{\circ} 7$.

Camacho Rosalía. (2003). Acercándonos a los instrumentos internacionales de protección de los derechos humanos de las mujeres. San José: Instituto Interamericano de Derechos Humanos.

Comisión Interamericana de Derechos Humanos. El camino hacia una democracia sustantiva: la participación política de las mujeres en las Américas. OEA/Ser.L/V/II.Doc.79.

Gaceta del Estado Plurinacional de Bolivia. Ley n. 243 contra el Acoso y Violencia Política hacia las Mujeres.

Instituto Interamericano de Derechos Humanos. Aula Virtual Interamericana. Curso auto formativo. Recursos para incorporar los derechos humanos de las mujeres, Unidad 1, lectura 2, Derechos del Hombre.

Ministerio de Justicia de Bolivia. (2015). Cedaw. Cómo enfrentar la discriminación contra la mujer en Bolivia. Observaciones al Estado Plurinacional de Bolivia-07/2015, onu Mujeres Bolivia, noviembre.

Ministerio de Justicia de Bolivia. (2016). Guía de actuación de promotoras comunitarias "Por una vida libre de violencia". SOLIDAR Suiza-Aos. Bolivia.

Ministerio de Justicia. 5. y $6 .^{\circ}$ Informe periódico del Estado Plurinacional de Bolivia presentado al Comité para la eliminación de la discriminación contra la mujer (CEDAW).

NACIONES UNIDAS. Lista de cuestiones y preguntas relativa a los informes periódicos quinto y sexto combinados del Estado Plurinacional de Bolivia. CEDAw/C/BOL/Q/5-6/Add.1.

Oficina del Alto Comisionado de los Derechos Humanos de las Naciones Unidas. (2004). Los derechos de la mujer son derechos humanos. Nueva York y Ginebra.

oIT. (2006). Promoviendo la igualdad de género, Convenios de la oIt y los derechos laborales de las mujeres. Ginebra.

ONU Mujeres y Parlamento Latinoamericano y Caribeño. Norma Marco para consolidar la democracia paritaria. 
onu Mujeres, América Latina y el Caribe. Datos de ACOBOL sobre los resultados de las elecciones de 2015.

ONU Mujeres. Guía estratégica, empoderamiento político de las mujeres: marco para una acción estratégica, América Latina y El Caribe (2014-2017).

ONU Mujeres. (2016). Informe Anual 2015-2016. Nueva York.

Organización de las Naciones Unidas. (2016). Agenda 2030 y los Objetivos de Desarrollo Sostenible, una oportunidad para América Latina y el Caribe.

Tribunal Electoral del Poder Judicial de la Federación. Protocolo para Atender la Violencia Política contra las Mujeres.

VÁsquez, R. (2013). El acoso y la violencia política hacia las mujeres en Bolivia. Avances formales y desafíos reales para la igualdad. Asociación de Concejalas de Bolivia АсовоL у Cooperación Alemana al Desarrollo - GIZ. 